\title{
Study on the Effective Time of Biological Effects of the Electromagnetic Field on Mung Bean Seed
}

\author{
Yaxiang Bai \\ School of Science, Dalian Ocean University \\ Dalian Ocean University \\ Liaoning Province, China \\ Byx0671@163.com \\ Bin Kang \\ School of Science, Dalian Ocean University \\ Dalian Ocean University \\ Liaoning Province, China \\ 499238181@qq.com
}

\author{
Yuan Huo \\ School of Science, Dalian Ocean University \\ Dalian Ocean University \\ Liaoning Province, China \\ 653785294@qq.com
}

\begin{abstract}
In order to further explore the biological effects of electromagnetic treatment technology on crop seeds, in this paper, the mung bean seeds are treated by electric and magnetic field with different dose, then mungbean seeds are cultivated in water, a control group is set. By observing the change of the circumstance of the germination percentage, the best intensity of the electric field and magnetic field are selected, so that this kind of technology can be well used in agriculture. The experiment shows that the germination percentage of seeds treated by electric field and magnetic field can be improved, and the seeds treated by electric field with $25 \mathrm{kV}$ and magnetic field with $0.6 \mathrm{~T}$ is superior to other groups on the germinate rate.
\end{abstract}

Keywords-Electric field; magnetic field; germination rate; mung bean seeds; biological effect

\section{INTRODUCTION}

In recent years the biological effect of high voltage static electric field and magnetic field has been attracting more and more attention, a great amount of work has been done on it by biologists and physicists, and a great deal of fruits have been reached. Many experiments results show that the electric field and magnetic field has obvious effect on the biology, especially the crops. For example, it was reported by Shenyang Agricultural University that the static electric fields $(40 \mathrm{kv} / \mathrm{cm} \times 12 \mathrm{~h})$ can make the yield of cotton increase by $12.4 \%$, the cotton length increases by $1 \sim 2 \mathrm{~mm}[1]$. While Professor Liang Yun-zhang found that if the beet seeds were stimulated by high voltage static electric field, then the amount of sugar of beet was enhanced by 0.6 degrees, the yield of mu by about $7 \%$ [2]. With advantages including simple equipment, easy operation, immediate processing, energy saving and environmental protection, the electric field method and magnetic field method are often used in the seed treatment. However, the effect of electric field and magnetic field seed processing technologies fail sometimes to be significantly reflected in practical application, leading to the incomplete adoptions of such two technologies. In the exploration and application process, it is found that the seeds processed by magnetic filed or electric field cannot immediately enter the status of bud, sowed and grow up.Instead, they should be put into storage for sale by seeds company and then purchased by farmers, which means the seeds processed by electric field or magnetic field must be stored for certain period of time before being sowed. The electric field or magnetic field seed processing technologies exert present biological effects on the seeds. When the processed seeds are about to be sowed, whether the biological effects still remained or not, in other words, whether there is a valid time about the biological effects of two seed processing technologies, is a long-lived issue needed to be focused and paid attention.

In this paper, experiments are conducted on the valid time of the effects of electric field or magnetic field upon the mung bean seeds, this research result is of vital significance to promote the theoretical and technological analys is of seed processing technology based on electric or magnetic fields.

\section{MATERIALS AND METHODS}

\section{A. The Determination of the Germination Rate}

Add water to the seeds at 9 o'clock every morning and record the germination number of the seeds, the length of the seeds' radicle reached around $1 \mathrm{~cm}$ can be regard as seed germination. 50 seeds in each group, calculate the germination rate.

Formula 1--1: germination $\operatorname{rate}(\%)=$ the germination number of the selected seeds / the number of selected seeds $* 100 \%$

\section{B. Experimental Materials}

1 GPJ-150 Light incubator(The temperature in the cabinet is set to $20{ }^{\circ} \mathrm{C}$ ), 1Model 7050 Electromagnet 
Power Supply (Pole width is $8 \mathrm{~cm}$ ), 1 ZGF-I Direct-current High Voltage Generator, several beakers, several petri dishes, several mung beans.

\section{Experimental Methods}

Use the uniformelectric field and magnetic field as the control variables to treat the mung bean seeds with different length of time. Put the treated seeds into the storage bags, divided them into equal portions randomly, and then place them respectively for 0 week, 1 week, 2 weeks, 3 weeks, 4 weeks and 5 weeks. To cultivate the treated seeds in accordance with their different placement time and change the water for the seeds at 9 am everyday. Starting from the cultivation with water to the germination of the seeds, the experimental period lasted for 7 whole days. Record the germination number of the seeds everyday, and calculate the germination rates.

\section{EXPERIMENT AL RESULTS AND ANALYSIS}

A. The Effects of the Electric Field on Mung Bean

\section{Seeds Germination}

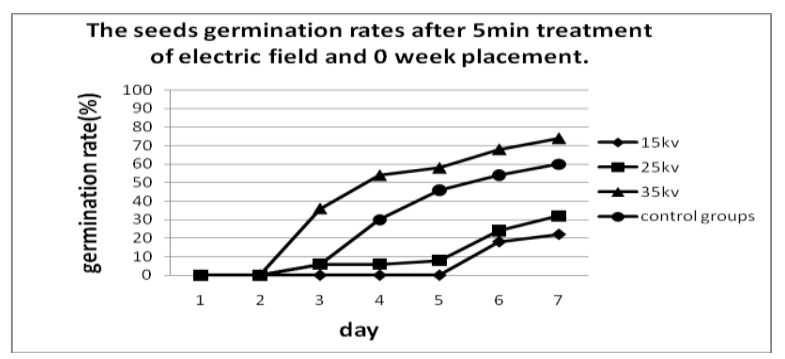

Figure 1. The seeds germination rates after 5 min treatment of electric field and 0 week placement

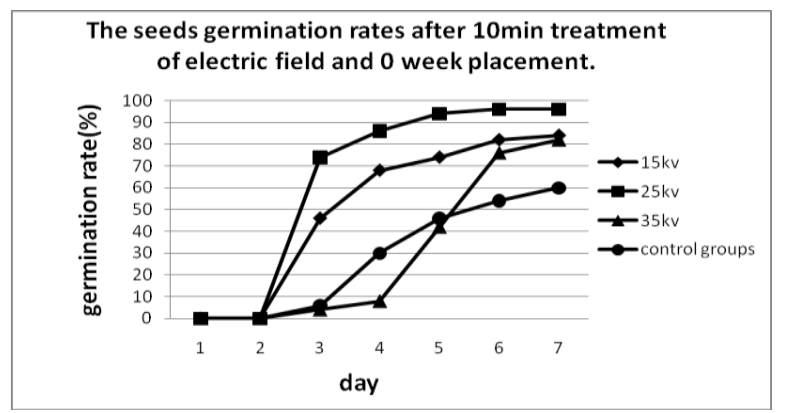

Figure 2. The seeds germination rates after $10 \mathrm{~min}$ treatment of electric field 0 week placement.

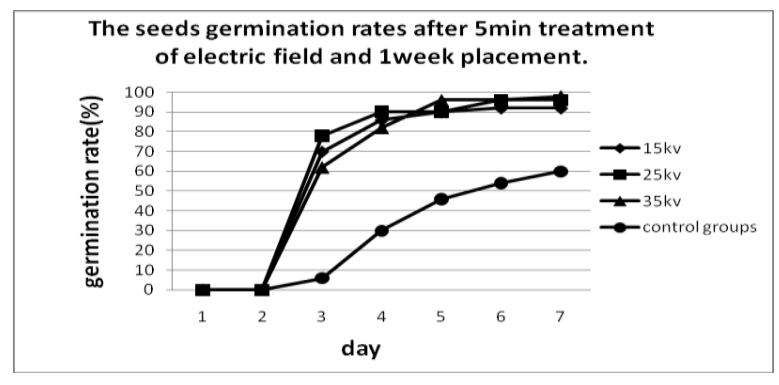

Figure 3. The seeds germination rates after $5 \mathrm{~min}$ treatment of electricfield and 1 week placement.

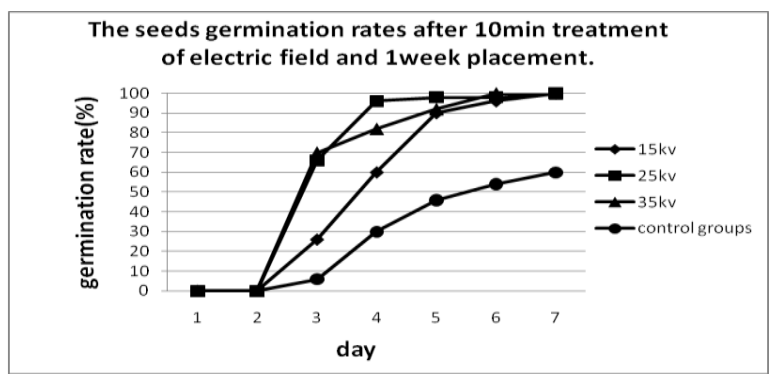

Figure 4. The seeds germination rates after $10 \mathrm{~min}$ treatment of electric field and 1 week placement.

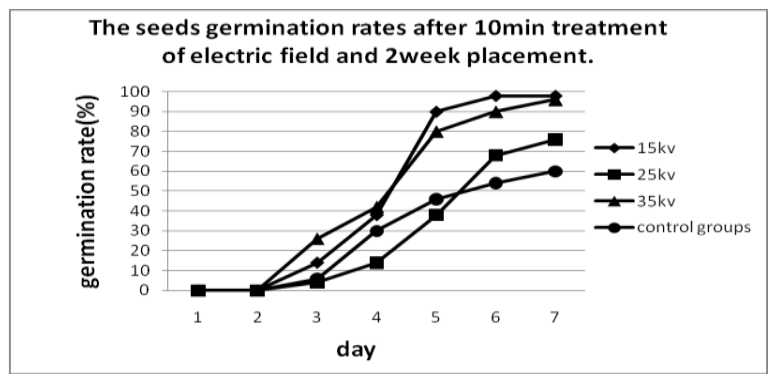

Figure 5. The seeds germination rates after 5 mintreatment of electric field and 2 weeks placement.

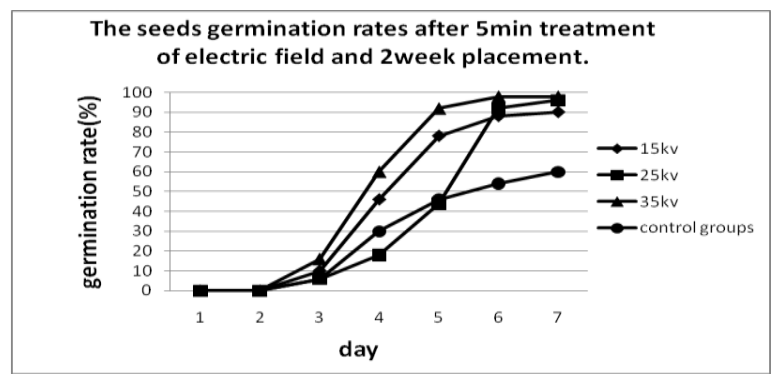

Figure 6. The seeds germination rates after $10 \mathrm{~min}$ treatment of electric field and 2 weeks placement. 


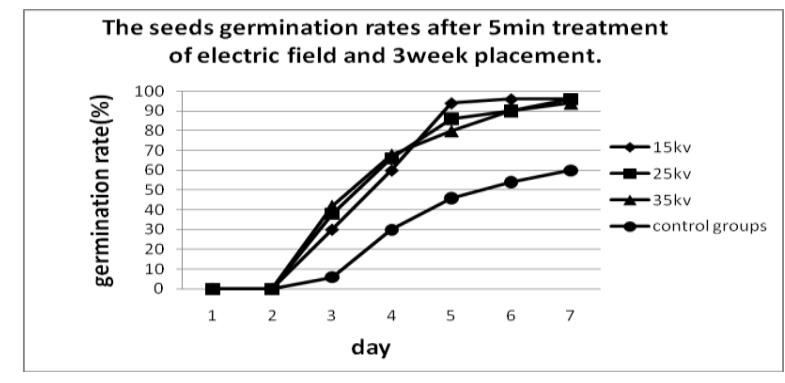

Figure 7. The seeds germination rates after $5 \mathrm{~min}$ treatment of electric field and 3 weeks placement.

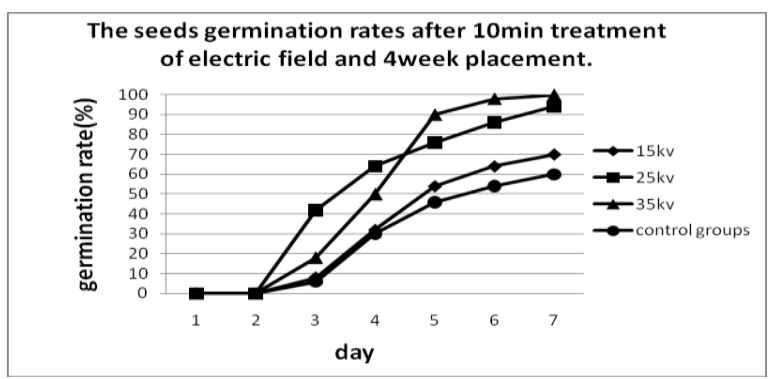

Figure 8. The seeds germination rates after $10 \mathrm{~min}$ treatment of electric field and 3 weeks placement.

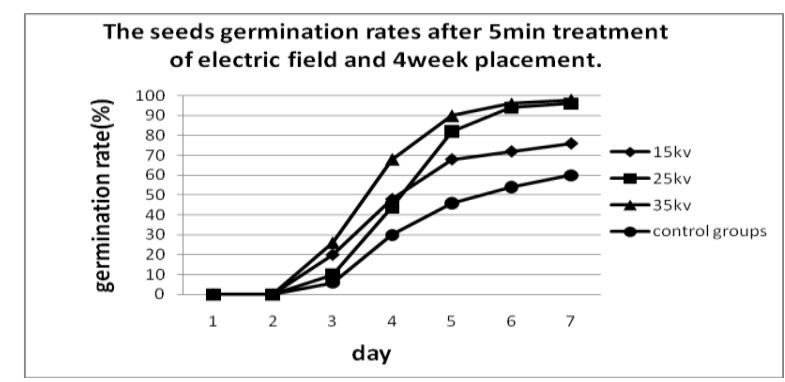

Figure 9. The seeds germination rates after 5 min treatment of electric

field and 4 weeks placement.

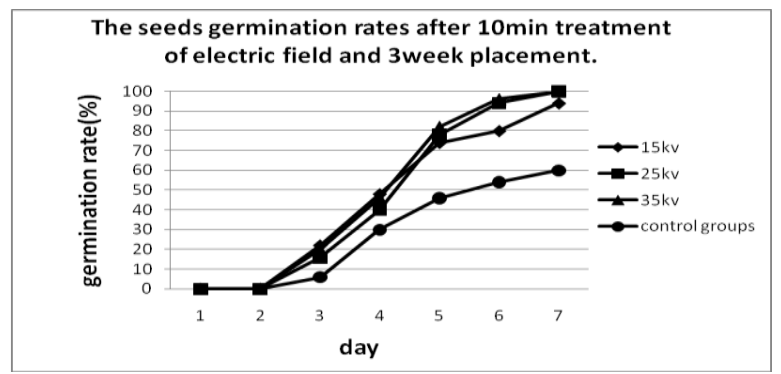

Figure 10. The seeds germination rates after 10 min treatment of electric field and 4 weeks placement.

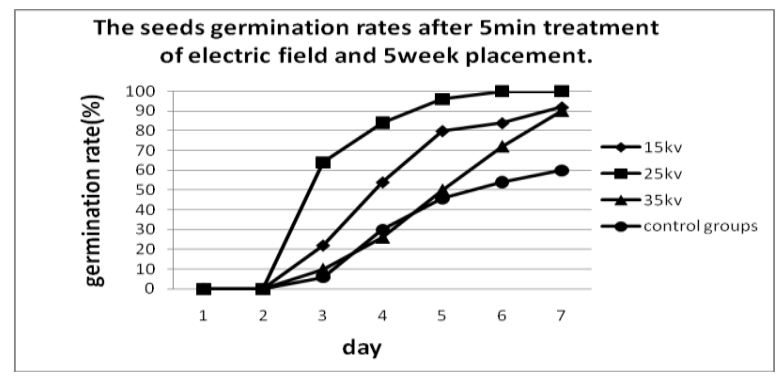

Figure 11. The seeds germination rates after 5 min treatment of electric field and 5 weeks placement.

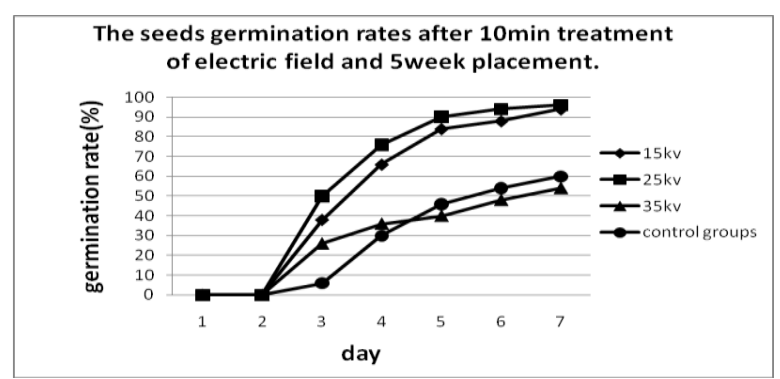

Figure 12. The seeds germination rates after $10 \mathrm{~min}$ treatment of electric field and 5 weeks placement.

From Fig. 1 to 12 , it can be seen that, after the treatment of the electric field, no matter how many weeks the seeds are placed, they all do not germinate during the first two days. From the third day, all the treated seeds start geminating and exceed the seeds in the control groups(In the 36 groups of treated seeds, only 6 groups' seeds germination rates are lower than the control groups'). The germination rates and germination speed of the treated seeds after 1, 3 and 4 weeks placement all exceed the control groups'. With the electric field as the variable, the electric field intensity of $35 \mathrm{kv} / \mathrm{m}$ win seven times in the 12 groups of data of the germination rates, this is enough to prove that, after the treatment of the electric field intensity of $35 \mathrm{~km} / \mathrm{m}$, the seeds germination rates are higher than the other groups'. With the treatment time as the variable, from the figures, it can be seen that the germination rates of the seeds with $5 \mathrm{~min}$ treat ment are higher than those with 10 min treatment.

B. The Effects of the Magnetic Field on Mung Bean

\section{Seeds Germination}

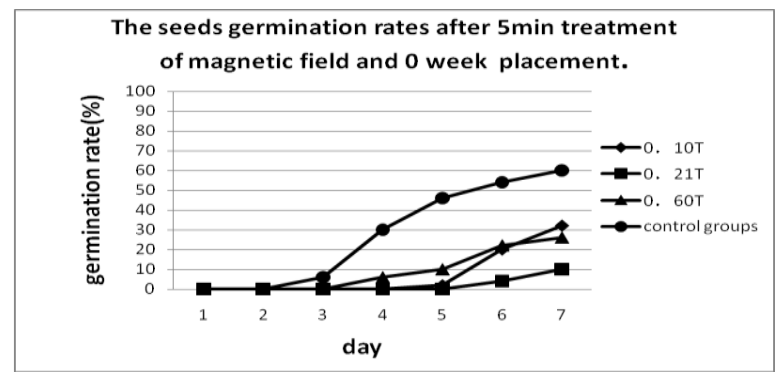

Figure 13. The seeds germination rates after 5 mintreatment of magnetic field and 0 week placement. 


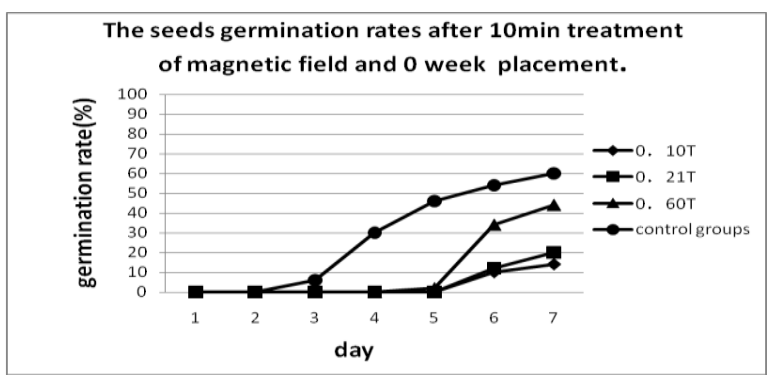

Figure 14. The seeds germination rates after $10 \mathrm{~min}$ treatment of magnetic field and 0 week placement.

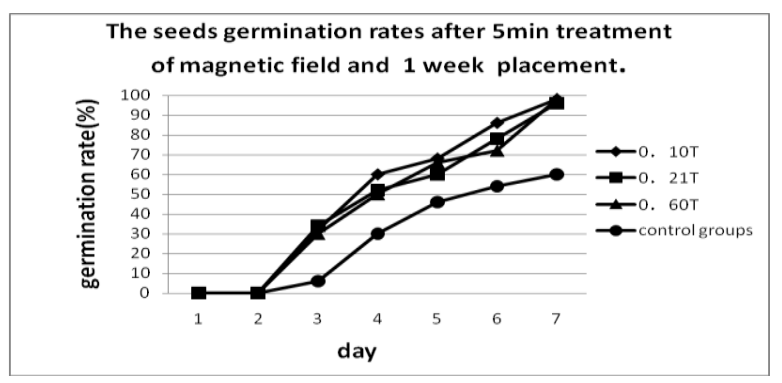

Figure 15. The seeds germination rates after 5 min treatment of magnetic field and 1 week placement.

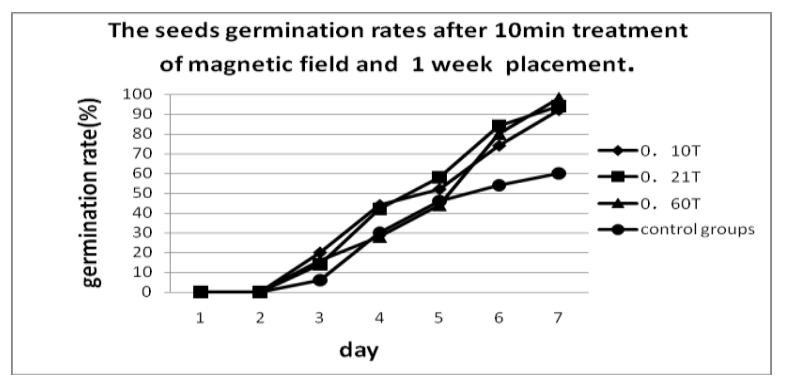

Figure 16. The seeds germination rates after 10 min treatment of

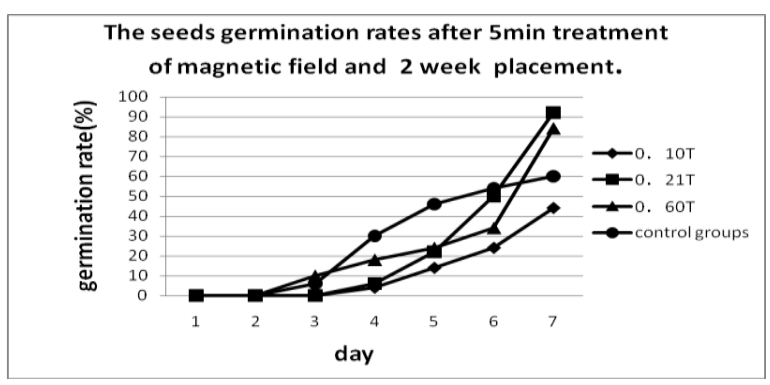

magnetic field and 1 week placement.

Figure 17. The seeds germination rates after 5 min treatment of magnetic field and 2 weeks placement.

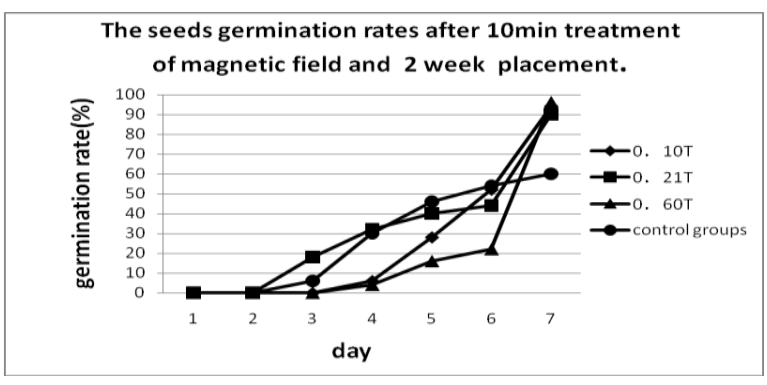

Figure 18. The seeds germination rates after $10 \mathrm{~min}$ treatment of magnetic field and 2 weeks placement.

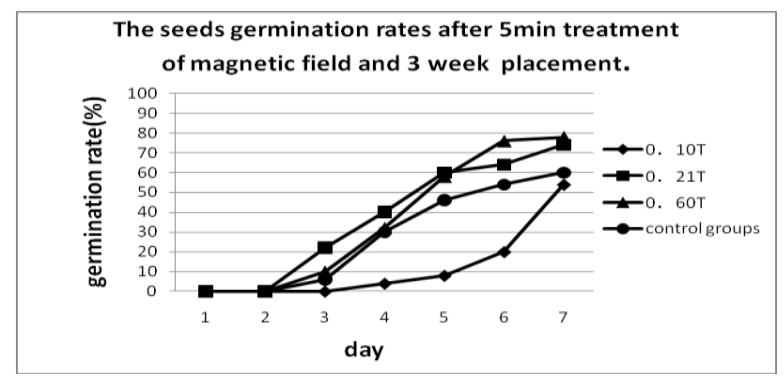

Figure 19. The seeds germination rates after 5 mintreatment of magnetic field and 3 weeks placement.

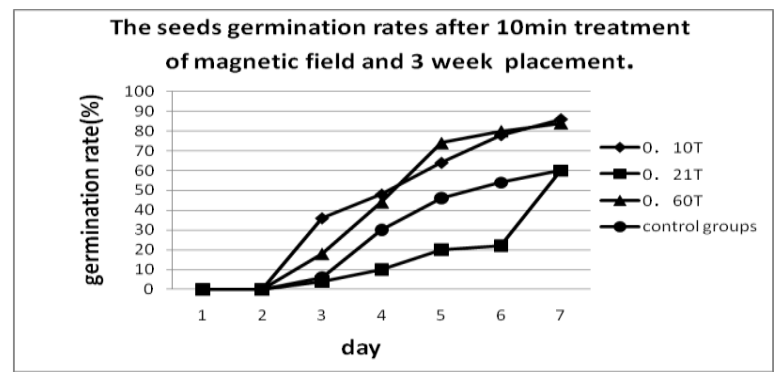

Figure 20. The seeds germination rates after 10min treatment of magnetic field and 3 weeks placement.

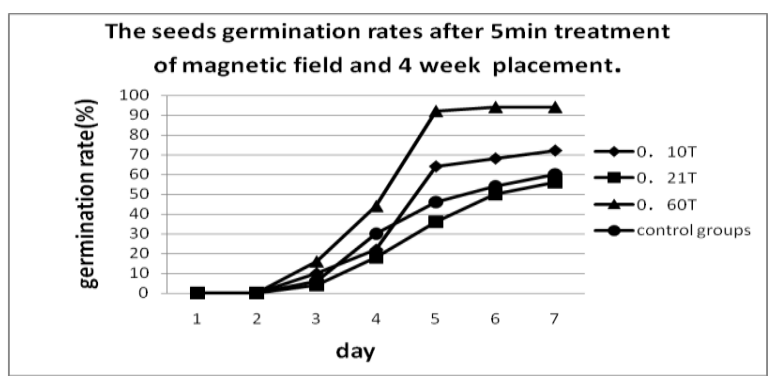

Figure 21. The seeds germination rates after 5 min treatment of magnetic field and 4 weeks placement. 


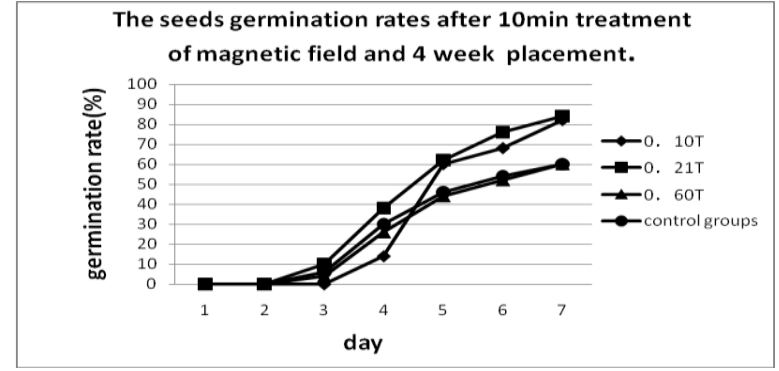

Figure 22. The seeds germination rates after $10 \mathrm{~min}$ treatment of magnetic field and 4 weeks placement.

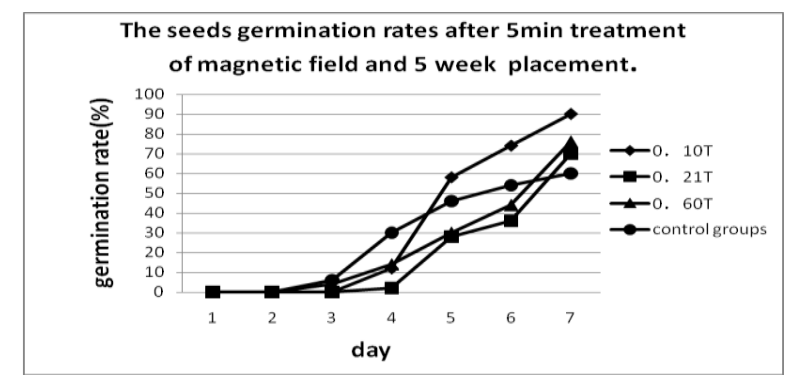

Figure 23. The seeds germination rates after 5 min treatment of magnetic field and 5 weeks placement.

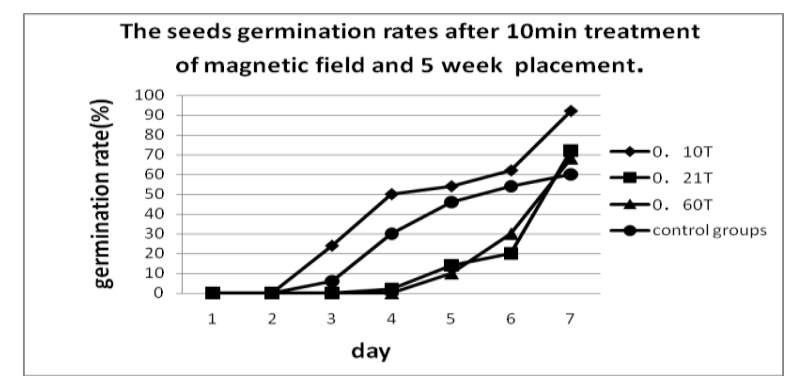

Figure 24. The seeds germination rates after $10 \mathrm{~min}$ treatment of

magneticfield and 5 weeks placement.

From Fig. 12 to 24, it can be seen that, after the treatment of the magnetic field, no matter how many weeks the seeds are placed, they all do not germinate during the first two days. From the third day the treated seeds start to germinate, but the germination rate of the seeds treated by the magnetic field is obviously lower than those treated by the electric field and there happens the situation that the germination rates of the seeds which directly be cutivated without placement are all lower than the control groups'. To treat the seeds after 1 week placement can gain the best results and all the 6 groups of data exceed the control groups'. To treat the seeds after 25 weeks placement, their germination rates fluctuate greatly and have no obvious regularity. After 25 weeks placement, the germination rates of all the magnetic field treated seeds may be lower than the control groups'. With the magnetic field as the variable, the intensity of $0.60 \mathrm{~T}$ performs strongly, 5 of the 12 groups of data gain the best results ( 2 of the 12 groups of data are lower than the control groups'). With the treatment time as the variable, the data of the seeds treated for $5 \mathrm{~min}$ has an obvious advantage.

\section{THE EXPERIMENT AL RESULTS AND DISCUSSIONS}

\section{A. Experimental Results}

The effects of the electric field on mung bean seeds are stronger than the magnetic field, the germination rate is the highest when the seeds are treated by the electric field with intensity of $35 \mathrm{kV} / \mathrm{m}$ for $10 \mathrm{~min}$ and placed for 1 week, that means the optimal dose of the electric field treatment for the seeds is around $35 \mathrm{kV} / \mathrm{m}$. The germination rate is the highest when the seeds is cultivated by water after treated by the magnetic field with intensity of $0.60 \mathrm{~T}$ for $10 \mathrm{~min}$ and placed for 1 week. The optimal dose of the magnetic field is around 0,60T and the treated seeds shouldn' $t$ be cultivated right away or after a long time placement, the optimal placement time is around 1 week.

Meanwhile, something special happens during the experiment. For example, after being treated by the electric field with intensity of $25 \mathrm{kV} / \mathrm{m}$ for $10 \mathrm{~min}$ and placed for 0 week, the seeds start to rot after the forth day. To cultivate the seeds after they are treated by the electric field with intensity of $35 \mathrm{kV} / \mathrm{m}$ for $10 \mathrm{~min}$, their germ reached a length of $3 \mathrm{~cm}$ on the third day, same thing happens when the seeds are treated by the electric field with intensity of $25 \mathrm{kV} / \mathrm{m}$ for $5 \mathrm{~min}$.

\section{B. Discussions on the Results}

The mechanism of treating the crop seeds with electromagnetic technology is quite a complex process. There is no exact conclusion on this so far, many researchers put forward lots of questions on the problem and they thought preliminarily that it has something to do with the following items:

The magnetic field impacts on the seeds of plants as a physical factor which can stimulate the information carried in the plant body, thus produce and promote the development of the plant body mechanism.

The magnetic field can enhance the inside enzyme activity of the crop seeds. The germination and growth of the crop seeds is a series of performance and process of enzymatic reaction, and the enzymatic reaction speed depends on the enzyme activity directly[3]. So the reason that the magnetic field can promote the seeds germination is because it can promote a series of enzyme reaction inside the seeds and make the seeds possess all kinds of nutrition consequently.

The magnetic field can accelerate the water absorbing process of the plant seeds. The magnetic treatment can change the quantity of the water which combined with the macromolecules in different degrees, the quantity of the free water and the degree of the hydration of the protein condensates in the cells. Thus the movement of the cytoplasm speed up and the condensed state, the viscosity and the water holding capacity of the cells changes [4].

The megnetic field can increase the respiration and other actions of the plant seeds.

The magnetic field can accelerate the absorptio$\mathrm{n}$ and utilization of the nutrients inside the plant seeds.

The biological effects of electromagnetic technology on plant seeds have a certain limit. It' $s$ not true that the stronger is the electromagnetic field intensity, the more 
obvious is the biological effects it impact on the seeds, but it plays a positive role within a certain intensity and a negative role beyond the certain intensity, which shows that the effects of electromagnetic technology on the plants are very complex and call for our further explorations and harder research in the future. What's more, different kinds of magnetic treatments lead to different results and the effects of the same kind of magnetic treatment also varies in different years[5].

\section{CONCLUSIONS AND SUGGESTIONS}

\section{A. Conclusions}

This experiment adopts different electromagnetic field intensity to treat the mung bean seeds and cultivates them with water after different time of placement. The germination rates are measured in the experiment and compared with the untreated groups. The following conclusions can be drawn after the research and analysis:

The effects of the electric field technology on mung bean seeds are very clear. The electric field promotes the germination of the seeds which makes the seeds gemination rates exceed the control groups' and its promotion effects become more and more obvious with the enhancement of the electric field intensity. The electric field not always have promoting effects, but how much electric intensity will have inhibiting effects has to be further studied.

The effects of the magnetic field technology on mung bean seeds are not very clear, the regularity of data obtained in the experiment is unconspicuous. But in general trend, the magnetic field performs promoting effects on the mung bean seeds within a certain range and further data analys is calls for further research.

Different magnetic field intensity may have different effects on the activity of the seeds. But comparing to other treatment methods, the magnetic treatment needs small amount of labor, it's cheaper and has little harm to operating person. Also it does not contaminate the soil, the environment ot the crops. The magnetic treatment is in favor of protecting the environment and has a broad utilization future on agricultural production[6].

\section{B. Suggestions}

Only three elements including different electromagnetic field intensity, treatment time and placement time are considered in the experiment due to the limitation of the funds, raw materials, time and so on, there are still some problems needing further studies and discussions. In order to have a better study and discussion on the relevant issues in the future, the author suggests that the research should be started from analyzing the mechanism of the influence of the biological effects of the electromagnetic technology on the seeds, so that the technology can be better used in the future.

\section{REFERENCES}

[1] Liu Fu-quan. The effect of the static electric field on cotton, Journal of Dongbei Agriculture University, 1974, 1: 86.

[2] Liang Yun-zhang, Biotic effects and application of high voltage static electric field, Physics, 1995, 1:39.

[3] GaoSheng Lu, Jin Yan Yu. The Biological Effects of Magnetic Field Treatment on Crop Seed and the Research Progress of Its Mechanism. Seed, 1990,(3):47-49

[4] YaLi Liu, ShuSong Yue, Liu Ling et al. The Physiological and Biochemical Effects of Magnetized Water on Crops. Journal of Henan Normal University(Natural Science), 2002, 30(3):82-84

[5] ShiMin He, GuoCheng Zhang. The Effects of Magnetized Water on Soybean Seed Germination Stage and the Anti-saline properties of the Seedling Stage. Journal of Science of Teachers' College and University, 2000, 20(1):41-44.

[6] QingFang Li, YongHong Fan, ChengCang Ma. The Changes of the Content of Protein, Fat and Starch in the Process of Soybean Seed Germination. Journal of Anhui Agricultural Sciences. 1988,4(9):34-35

[7] GuoLin Zhao, Cao Hong, GuoZhu Zhang et al. The Research on Wheat Physiological Metabolism and yield-improving effects of Magnetized Water Soaked Seeds. Acta Agriculturae BorealiOccidentalis Sinica, 1997,6(4):42-46. 